\title{
Predictors of Thirty-Day Readmission Among Hospitalized Family Medicine Patients
}

\author{
Gregory M. Garrison, MD, MS, Meghna P. Mansukhani, MBBS, \\ and Bradley Bohn, MD
}

Purpose: Hospital readmissions within 30 days of initial discharge occur frequently. In studies of elderly patients receiving Medicare, readmissions have been associated with poor-quality inpatient care, ineffective hospital-to-home transitions, patient characteristics, disease burden, and socioeconomic status. Among adult family medicine patients spanning a wide age range, we hypothesize that previous hospitalizations, length of stay, number of discharge medications, medical comorbidities, and patient demographics are associated with a greater risk of hospital readmission within 30 days.

Methods: A retrospective case-control study of 276 family medicine inpatients was conducted to determine the factors associated with 30-day readmission. Bivariate statistics were computed and a multivariate analysis using logistic regression was performed to determine the independent effects of each factor.

Results: Patients readmitted within 30 days had more hospitalizations, more emergency department visits, longer hospital stays, more comorbidities, and more discharge medications and were less likely to be married. Multivariate logistic regression found that hospitalization within the previous 12 months (odds ratio, 2.71) and long hospital stays (odds ratio, 2.16) were associated with 30-day readmission; being married (odds ratio, 0.54 ) had a protective effect.

Conclusions: This study demonstrates that factors previously found to be associated with 30-day readmission among elderly patients receiving Medicare also apply to family medicine patients of all ages. It also demonstrates prior hospitalizations, length of stay, and marital status are useful proxies for many more complicated factors, such as disease burden, medical complexity, and social issues, that influence hospital readmission. (J Am Board Fam Med 2013;26:71-77.)

Keywords: Family Medicine, Logistic Models, Patient Readmission, Risk Factors

Hospital readmission within 30 days of discharge occurs in almost $20 \%$ of patients receiving US Medicare, costing an estimated $\$ 17.4$ billion in $2004 .{ }^{1}$ Unplanned readmissions may be a marker of poor-

This article was externally peer reviewed.

Submitted 24 April 2012; revised 26 September 2012; accepted 8 October 2012.

From the Department of Family Medicine, Mayo Clinic, Rochester, MN (GMG BB); and Affiliated Community Medical Centers, Willmar, MN (MPM).

Funding: Support for this study was provided by the Department of Family Medicine, Mayo Clinic, Rochester, MN

Prior Presentation: This work was presented as a poster, "Avoiding Hospital Readmission: Using a Predictive Model to Drive Coordinated Care," at the Society of Teachers of Family Medicine Conference on Practice Improvement, San Antonio TX, Dec 2-5, 2010.

Conflict of interest: none declared.

Corresponding author: Gregory M. Garrison, MD, MS, 200 First Street SW, Rochester, MN 55905 (E-mail garrison.gregory@mayo.edu). quality inpatient care ${ }^{2-4}$ or ineffective hospital-tohome transitions. ${ }^{5,6}$ However, certain patient characteristics, disease characteristics, and socioeconomic factors also are associated with increased risk of hospital readmission. ${ }^{1,7-11}$ Many of the studies identifying these factors were performed with elderly populations receiving Medicare ${ }^{1,7-9,11,12}$ and may not be applicable to younger patients. Other studies looking at hospital readmissions are disease specific. ${ }^{13-21}$ There are relatively few studies examining the risk factors for readmission among all primary care patients, and many of these were performed outside the United States or in hospitalist practices. ${ }^{10,22-25}$

It is well known that a small proportion of patients account for a disproportionate number of hospital admissions and health care costs. ${ }^{26-28}$ Identifying these patients is important for quality of care and economic reasons. ${ }^{29}$ This study determines the fac- 
tors present at the initial discharge that are associated with 30-day readmission for adult patients of all ages who are admitted to a family medicine inpatient service.

\section{Methods}

Factors such as previous hospitalizations, length of stay, number of discharge medications, and medical comorbidities were hypothesized to be associated with a greater risk of hospital readmission within 30 days, the dependent variable. These factors were chosen because in general they are easily obtained within electronic medical records and they have been shown to predict initial hospitalization in elderly patients older than age $60 .^{29}$

Sample size was calculated based on detecting a single-variable odds ratio of at least 2.0 , with $80 \%$ power at a significance level of .05. Prior hospitalization was presumed to be the dominate independent variable, ${ }^{8,29}$ and the sample size was based on this factor. Using an estimated proportion of exposed controls of $30 \%$ (for prior hospitalization) and a ratio of controls to cases of 2:1, approximately 100 cases and 200 controls would be needed. The year before this study, the family medicine service admitted 1234 patients, with a 30-day readmission rate of $11.9 \%$ (147 patients). Thus, a 1-year time frame was predicted to provide sufficient cases and controls for the study.

A retrospective case-control study of 276 patients admitted to a family medicine inpatient service serving community patients in Olmsted and Dodge Counties in Minnesota over a 12-month period-from November 1, 2008, through October 31, 2009-was conducted. Cases were patients initially admitted to the family medicine inpatient service and subsequently readmitted within 30 days of discharge to any inpatient hospital service. Controls were patients admitted to the family medicine inpatient service and not readmitted to any inpatient service within 30 days of discharge. An inpatient registry was used to identify potential cases and controls. Of 1164 admissions during the study period, 132 admissions were found to have been readmitted to the family medicine service within 30 days. Only the first admission of each patient during the study period was considered, thus eliminating 34 multiple readmissions. After excluding 2 women who were pregnant during the study period and 9 patients who lacked research authorization,
87 potential cases were left. Of the 1032 remaining cases that were not readmitted within 30 days, 198 were chosen at random from the portion who had granted research authorization. After excluding 3 who died within 30days of discharge and 6 who were pregnant during the study period, 189 potential control patients remained.

Chart review found 8 of the 189 potential controls to have been readmitted to another inpatient service at the institution within 30 days of discharge. These were reclassified as cases. Because of the structure of the inpatient registry, there was no way to initially distinguish patients who transferred to another service such as cardiology, the intensive care unit, or general surgery temporarily during their hospital stay. We found 11 instances of such service transfers among the 87 potential cases. Because these were not readmissions, they were reclassified as controls. It is unlikely that any of the controls sought care at other institutions because this family medicine service only cares for local patients who have an established primary care physician at the institution. Figure 1 shows how the 84 cases and 192 controls were established.

For each subject, data were abstracted into a spreadsheet regarding care before hospitalization, hospital course, number of medications (including over-the-counter medications), follow-up care, medical comorbidities, and demographics (Table 1). Data analysis was performed using $\mathrm{R}$ version 2.4.0 (http://www.r-project.org/) running on Mac OS 10.5 (Apple, Inc., Cupertino, CA). Bivariate statistics for the various factors were computed using a Wilcoxon rank sum test for numerical data and Fisher exact test or $\chi^{2}$ test for categorical data. $P$ values $<.05$ were considered significant. A multivariate analysis using logistic regression techniques was performed to determine the effects of each factor on the dependent variable (readmission within 30 days). This allowed computation of odds ratios with $95 \%$ confidence intervals for the various factors.

The protocol was approved by the institutional review board. All subjects signed research authorization that was kept on file, allowing retrospective chart review.

\section{Results}

As shown in Table 1, there were no differences in age, sex, education level, or living situation among 
Figure 1. Selection of cases and controls.

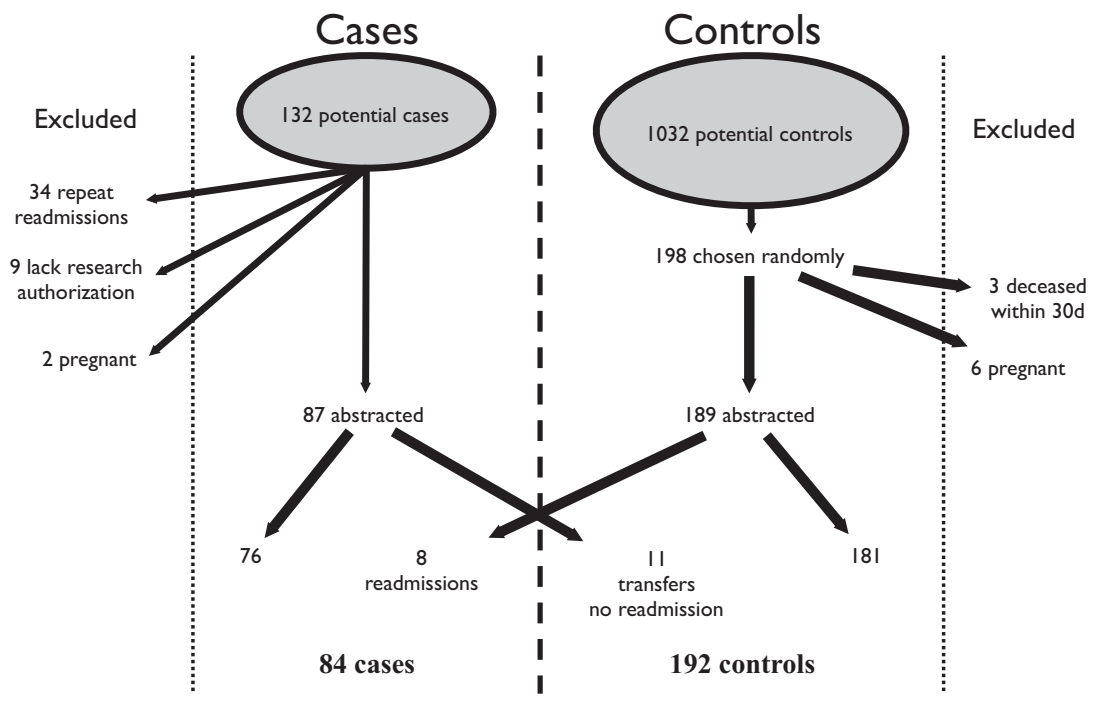

the 84 patients readmitted within 30 days (cases) and the 192 patients not readmitted within 30 days (controls). Patients readmitted within 30 days were less likely to be married than controls $(44.0 \%$ vs $57.8 \% ; P=.037)$.

Patients readmitted within 30 days averaged more hospitalizations over the past 12 months (2.32 vs $0.60 ; P<.0001)$ and had more emergency department visits over the past 12 months (4.12 vs 2.03; $P<.0001)$ than controls. Two-thirds of patients readmitted within 30 days had at least one hospitalization in the past 12 months, whereas twothirds of controls had no hospitalizations in the past 12 months. Length of stay was longer for those readmitted within 30 days ( 3.5 vs 2.3 days; $P=$ $.0422)$, and they were discharged while taking more medications (13.2 vs $10.2 ; P=.0006$ ). Patients readmitted within 30 days had more comorbidities ( 2.1 vs $1.2 ; P<.0001$ ), and the individual comorbidities of coronary artery disease, congestive heart failure, chronic obstructive pulmonary disease, recent cancer, and presence of a psychiatric disorder were all more common among cases.

Figure 2 shows the distribution of ages, previous hospitalizations, length of stay, and medications being taken at discharge for both cases and controls using a box and whiskers plot. This type of plot graphically shows 5 statistical measures: the extremes, the upper and lower quartiles, and the median. $^{30}$

Table 2 shows the results of the multivariate analysis. Readmission within 30 days was the dependent variable. Continuous independent variables such as number of medications being taken at discharge, length of stay, and number of hospitalizations were converted to dichotomous variables by selecting clinically significant thresholds. Thresholds of $\geq 10$ discharge medications, $>3$-day hospital stay, and one or more prior hospitalizations were chosen after reviewing the distributions as shown in Figure 2 and consulting published literature using similar cutoff values. ${ }^{1,17,23,24,29}$ This was done to enable the computation of an easily interpretable odds ratio for all independent variables. We kept prior hospitalizations and age as primary independent variables because of our hypothesis. Other factors that were not found by univariate analysis to be significant and those that were highly correlated with another factor (eg, prior emergency department visits and prior hospitalizations) were dropped to preserve the power of the model within a limited sample size.

Being married (odds ratio [OR], 0.54; 95\% CI, $0.30-0.97$ ) was shown to have a protective effect on the odds of readmission within 30 days. Conversely, any hospitalizations within the past 12 months (OR, 2.71, 95\% CI, 1.49-4.95) and a lengthy hospital stay of $>3$ days (OR, 2.16; $95 \%$ CI, 1.11-4.21) were found to increase the odds of readmission within 30 days. The remaining factors of age, polypharmacy, nursing home discharge, and the individual comorbidities did not reach statistical significance. 


\begin{tabular}{|c|c|c|c|c|}
\hline & $\begin{array}{l}\text { Not Readmitted Within } \\
30 \text { Days }(\mathrm{n}=192)\end{array}$ & $\begin{array}{l}\text { Readmitted Within } \\
30 \text { Days }(\mathrm{n}=84)\end{array}$ & $\begin{array}{l}\text { Odds Ratio } \\
(95 \% \mathrm{CI})\end{array}$ & $P$ \\
\hline Age, mean years (SD) & $60.8(22.4)$ & $64.7(20.4)$ & & .2350 \\
\hline $18-50$ & $63(32.8)$ & $19(22.6)$ & & \\
\hline $51-64$ & $34(17.7)$ & $18(21.4)$ & & \\
\hline $65-79$ & $38(19.8)$ & $20(23.8)$ & & \\
\hline$\geq 80$ & $57(29.7)$ & $27(32.1)$ & & \\
\hline Sex & & & & .6021 \\
\hline Female & $103(53.6)$ & $48(57.1)$ & & \\
\hline Male & $89(46.4)$ & $36(42.9)$ & $0.87(0.50-1.50)$ & \\
\hline Marital status & & & & .0369 \\
\hline Single, widowed, or divorced & $81(42.2)$ & $47(56.0)$ & & \\
\hline Married & $111(57.8)$ & $37(44.0)$ & $0.58(0.33-0.995)$ & \\
\hline Education level, mean years (SD) & $13.1(2.55)$ & $12.6(2.24)$ & & .1372 \\
\hline Living situation & & & & 1.0000 \\
\hline Skilled care, assisted living & $39(20.3)$ & $17(20.2)$ & & \\
\hline Home, apartment & $153(79.7)$ & $67(79.8)$ & $1.00(0.51-2.03)$ & \\
\hline Hospitalizations in past 12 months, mean (SD) & $0.60(1.02)$ & $2.32(2.61)$ & & $<.0001$ \\
\hline$\geq 1$ Hospitalization in past 12 months & $67(34.9)$ & $56(66.7)$ & $3.71(2.10-6.68)$ & $<.0001$ \\
\hline ED visits in previous 12 months, mean (SD) & $2.03(3.00)$ & $4.12(4.17)$ & & $<.0001$ \\
\hline Length of stay (days), mean (SD) & $2.34(2.25)$ & $3.50(4.24)$ & & .0422 \\
\hline$>3$-Day hospital stay & $34(17.7)$ & $27(32.1)$ & $2.19(1.16-4.12)$ & .0113 \\
\hline Discharge medications, mean (SD) & $10.2(6.22)$ & $13.2(6.80)$ & & .0006 \\
\hline$\geq 10$ Discharge medications & $99(51.6)$ & $60(71.4)$ & $2.34(1.31-4.27)$ & .0023 \\
\hline ICU stay & $14(7.3)$ & $2(2.4)$ & $0.31(0.03-1.40)$ & .1606 \\
\hline Discharge to skilled nursing home & $28(14.6)$ & $12(14.3)$ & $0.98(0.43-2.12)$ & 1.0000 \\
\hline Comorbidities, mean (SD) & $1.2(1.44)$ & $2.1(1.67)$ & & $<.0001$ \\
\hline \multicolumn{5}{|l|}{ Comorbidities* } \\
\hline Coronary artery disease $(410-414)$ & $30(15.6)$ & $23(27.4)$ & $2.03(1.04-3.94)$ & .0303 \\
\hline Congestive heart failure (428) & $21(10.9)$ & $18(21.4)$ & $2.21(1.04-4.68)$ & .0252 \\
\hline Atrial fibrillation (427.3) & $19(9.9)$ & $11(13.1)$ & $1.37(0.56-3.68)$ & .5285 \\
\hline Diabetes (249-250) & $29(15.1)$ & $21(25.0)$ & $1.87(0.94-3.33)$ & .0615 \\
\hline $\begin{array}{l}\text { Chronic obstructive pulmonary disease } \\
\quad(491-492,496)\end{array}$ & $34(17.7)$ & $25(29.8)$ & $1.96(1.03-3.72)$ & .0372 \\
\hline Chronic kidney disease $(582,585-586,588)$ & $27(14.1)$ & $20(23.8)$ & $1.90(0.94-3.81)$ & .0560 \\
\hline Cancer (140-209) & $15(7.8)$ & $14(16.7)$ & $2.35(1.00-5.53)$ & .0335 \\
\hline Psychiatric (291-292, 294-304, 306-312) & $63(32.8)$ & $41(48.8)$ & $1.95(1.12-3.40)$ & .0150 \\
\hline Attended follow-up visit & $151(78.6)$ & $69(83.1)$ & $1.34(0.66-2.84)$ & .4177 \\
\hline
\end{tabular}

For continuous values, the mean and (standard deviation) are reported. All other values are reported as n (\%). Bolded values are statistically significant $(P<.5)$.

*ICD-9 codes used to define comorbidity are listed in parenthesis.

ED, emergency department; ICU, intensive care unit.

\section{Discussion}

Prior hospitalizations and lengthy hospital stays increased the odds of readmission in our multivariate model. With an overall mean age of 62 years and a median of 66 years, nearly half the patients in our study were younger than those in the Medicare population that is typically studied; this is seen in Table 1 and Figure 2. Thus, our results extend and confirm previous work that has reported prior hos- pitalizations ${ }^{1,8}$ and length of stay ${ }^{1}$ as risk factors for readmission in elderly patients receiving Medicare.

Being married had a protective effect, reducing the odds of readmission in the multivariate model. Previous work has established a link between living alone and increased odds of readmission among elderly patients. ${ }^{9}$ However, marital status has not been looked at extensively and has yielded conflicting results. ${ }^{24,29}$ Being married likely reflects greater 
Figure 2. Distributions of age, previous hospitalizations, length of stay, and discharge medications between readmission cases and controls.
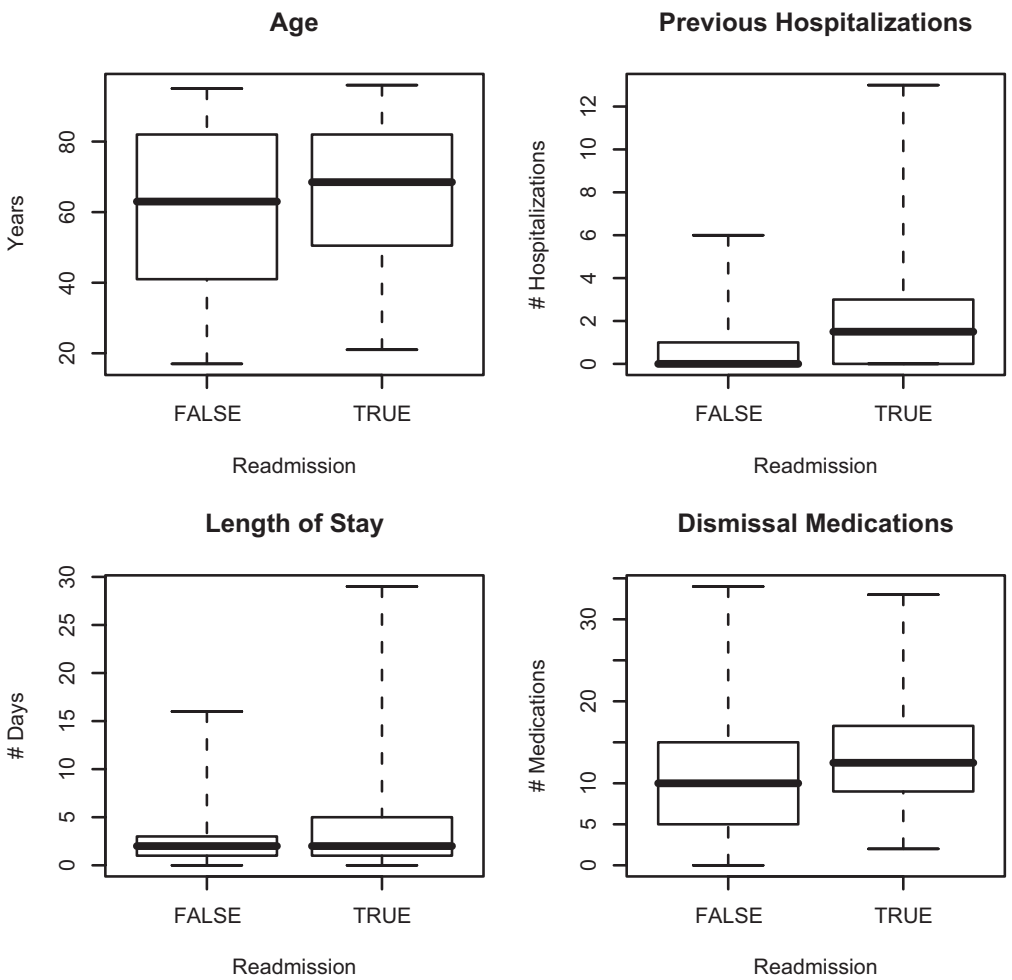

social support and not living alone. This study found it to be an important protective factor that should be studied further.

Previous studies of the Medicare population have demonstrated higher odds of readmission among the extreme elderly (older than age 80) when compared with those 65 to 70 years old, but these studies did not look at many patients younger than age $65.1,7,8$ Our multivariate results indicate that

patients younger than age 65 are just as likely to be readmitted as those older than age 65 when other variables are controlled.

The multivariate results did not find a significant link between specific comorbid conditions, number of comorbid conditions, or number of medications taken at discharge and odds of readmission. Previous studies have shown associations between specific disease conditions and odds of readmission. ${ }^{1,7}$ This was

Table 2. Results of Logistic Regression

\begin{tabular}{lccc}
\hline & Odds Ratio & $95 \%$ CI & P $(z)$ \\
\hline Age $\geq 65$ years & 0.71 & $(0.35-1.43)$ & 0.3451 \\
Married & 0.54 & $(0.30-0.97)$ & $\mathbf{0 . 0 3 9 8}$ \\
$\geq 1$ Hospitalization in past 12 months & 2.71 & $(1.49-4.95)$ & $\mathbf{0 . 0 0 1 1}$ \\
$\geq 10$ Discharge medications & 1.73 & $(0.86-3.53)$ & 0.1256 \\
$>$ 3-Day stay & 2.16 & $(1.11-4.21)$ & $\mathbf{0 . 0 2 3 2}$ \\
Discharge to nursing home & 0.63 & $(0.25-1.50)$ & 0.3038 \\
Coronary artery disease & 1.80 & $(0.86-3.76)$ & 0.1165 \\
Congestive heart failure & 1.28 & $(0.56-2.89)$ & 0.5503 \\
Chronic obstructive pulmonary disease & 1.27 & $(0.62-2.58)$ & 0.5085 \\
Cancer & 1.78 & $(0.73-4.33)$ & 0.2040 \\
Psychiatric & 1.10 & $(0.60-2.03)$ & 0.7492 \\
\hline
\end{tabular}

Bolded values are statistically significant $(P<.05)$. 
apparent in our bivariate analysis for coronary artery disease, congestive heart failure, chronic obstructive pulmonary disease, cancer, and psychiatric illness. But this association disappeared in the multivariate analysis once other factors such as prior hospitalizations and length of stay were considered. Some previous studies reporting disease associations with odds of readmission did not consider prior hospitalizations in their multivariate analyses ${ }^{7}$; when prior hospitalizations were accounted for, the association disappeared. ${ }^{8}$ It is logical that medically complicated patients with multiple comorbidities and therefore multiple medications being taken at discharge have more hospitalizations and longer hospital stays. Many disease-specific models fail to account for the multiple comorbidity often witnessed in hospitalized general medical patients. ${ }^{13-21}$ It is fortunate that increased hospitalization and lengthy hospital stays are useful proxies for disease severity and multiple comorbidities, which are more difficult to quantify.

Recent work has shown an association between regional rates of readmission and overall admission rates, underscoring the importance of regional utilization patterns in determining readmission rates. ${ }^{31}$ Our work suggests that a tendency toward individual hospital utilization, as measured by previous hospitalizations, also plays an important role in 30-day readmissions. Further study is warranted to determine whether higher outpatient utilization also is associated with readmission.

This study had a low dropout rate because most patients granted authorization for retrospective review of their medical records. All subjects had a primary care physician at the institution where the study was conducted, thus minimizing the chance of hospitalization at another institution. A longitudinal electronic medical record automatically notifies outpatient primary care physicians of their patient's hospital admission and allows real-time access to hospital records including laboratory tests, daily progress notes, consults, and the discharge summary. All patients were scheduled follow-up visit with their primary physician at the time of hospital discharge. Attendance at this visit was high and not associated with readmission. Thus, many issues surrounding transitions of care such as lack of discharge information, ${ }^{32}$ inaccurate medication lists, ${ }^{33}$ and poor outpatient follow-up ${ }^{1}$ are not as apparent in our study.

This initial study is limited by small sample size at a single institution. Although the institution is an academic tertiary care center, the patients studied were community residents who received primary care through the Department of Family Medicine and were admitted as inpatients on the family medicine service. Additional study is needed to validate the findings in typical family medicine inpatients at nonacademic hospitals.

\section{Conclusions}

Our study shows that prior hospitalizations, length of stay, and marital status become useful surrogates for many individual patient factors influencing hospital readmission that are much harder to enumerate and quantify. Primary care practices embracing the medical home concept can use this simple, readily available information to identify patients at high risk of hospital readmission, possibly targeting them for more intensive care management.

We appreciate the assistance of Ms. Julie Maxson in abstracting and collecting the data on which this study is based.

\section{References}

1. Jencks SF, Williams MV, Coleman EA. Rehospitalizations among patients in the Medicare fee-for-service program. New Eng J Med 2009;360:1418-28.

2. Ashton CM, Kuykendall DH, Johnson ML, Wray $\mathrm{NP}, \mathrm{Wu} \mathrm{L}$. The association between the quality of inpatient care and early readmission. Ann Intern Med 1995;122:415-21.

3. Balla U, Malnick S, Schattner A. Early readmissions to the department of medicine as a screening tool for monitoring quality of care problems. Medicine 2008; 87:294-300.

4. Halfon P, Eggli Y, Prêtre-Rohrbach I, Meylan D, Marazzi A, Burnand B. Validation of the potentially avoidable hospital readmission rate as a routine indicator of the quality of hospital care. Med Care 2006;44:972-81.

5. Balaban RB, Weissman JS, Samuel PA, Woolhandler S. Redefining and redesigning hospital discharge to enhance patient care: a randomized controlled study. J Gen Intern Med 2008;23:1228-33.

6. Coleman EA, Parry C, Chalmers S, Min SJ. The care transitions intervention: results of a randomized controlled trial. Arch Intern Med 2006;166:1822-8.

7. Silverstein MD, et al., Risk factors for 30-day hospital readmission in patients $\geq 65$ years of age. Proc (Bayl Univ Med Cent) 2008;21:363-72.

8. Marcantonio ER, McKean S, Goldfinger M, Kleefield S, Yurkofsky M, Brennan TA. Factors associated with unplanned hospital readmission among patients 65 years of age and older in a Medicare managed care plan. Am J Med 1999;107:13-7. 
9. Arbaje AI, Wolff JL, Yu Q, Powe NR, Anderson GF, Boult C. Postdischarge environmental and socioeconomic factors and the likelihood of early hospital readmission among community-dwelling Medicare beneficiaries. Gerontologist 2008;48:495-504.

10. Billings J, Dixon J, Mijanovich T, Wennberg D. Case finding for patients at risk of readmission to hospital: development of algorithm to identify high risk patients. BMJ. 2006;333:327.

11. López-Aguilà S, Contel JC, Farré J, Campuzano JL, Rajmil L. Predictive model for emergency hospital admission and 6-month readmission. Am J Manag Care 2011;17:e348-57.

12. Garcia-Pérez L, Linertová R, Lorenzo-Riera A, Vázquez-Díaz JR, Duque-González B, Sarría-Santamera A. Risk factors for hospital readmissions in elderly patients: a systematic review. QJM 2011;104: 639-51.

13. Phillips CO, Wright SM, Kern DE, Singa RM, Shepperd S, Rubin HR. Comprehensive discharge planning with postdischarge support for older patients with congestive heart failure: a meta-analysis. JAMA 2004;291:1358-67.

14. Hernandez AF, Greiner MA, Fonarow GC, et al. Relationship between early physician follow-up and 30day readmission among Medicare beneficiaries hospitalized for heart failure. JAMA 2010;303:1716-22.

15. Lau AC, Yam LY, Poon E. Hospital re-admission in patients with acute exacerbation of chronic obstructive pulmonary disease. Respir Med 2001;95:876-84.

16. Bahadori K, FitzGerald JM. Risk factors of hospitalization and readmission of patients with COPD exacerbation-systematic review. Int J Chron Obstruct Pulmon Dis 2007;2:241-51.

17. Khawaja FJ, Shah ND, Lennon RJ, et al. Factors associated with 30-day readmission rates after percutaneous coronary intervention. Arch Intern Med 2012;172:112-7.

18. Hannan EL, Zhong Y, Krumholz H, et al. 30-day readmission for patients undergoing percutaneous coronary interventions in New York state. JACC Cardiovasc Interv 2011;4:1335-42.

19. Betihavas V, Davidson PM, Newton PJ, Frost SA, Macdonald PS, Stewart S. What are the factors in risk prediction models for rehospitalisation for adults with chronic heart failure? Aust Crit Care 2012;25: 31-40.

20. Bowersox NW, Saunders SM, Berger BD. Predictors of rehospitalization in high-utilizing patients in the VA psychiatric medical system. Psychiatr Q 2012;83:53-64.

21. McGhan R, Radcliff T, Fish R, Sutherland ER, Welsh C, Make B. Predictors of rehospitalization and death after a severe exacerbation of COPD. Chest 2007;132:1748-55.

22. Yam CH, Wong EL, Chan FW. Avoidable readmission in Hong Kong-system, clinician, patient or social factor? BMC Health Serv Res 2010;10:311.

23. van Walraven C, Dhalla IA, Bell C, et al. Derivation and validation of an index to predict early death or unplanned readmission after discharge from hospital to the community. CMAJ 2010;182:551-7.

24. Hasan O, Meltzer DO, Shaykevich SA, et al. Hospital readmission in general medicine patients: a prediction model. J Gen Intern Med 2010;25:211-9.

25. Lee EW. Selecting the best prediction model for readmission. J Prev Med Public Health 2012;45: 259-66.

26. Sommers A, Cohen M. Medicaid's high cost enrollees: how much do they drive program spending? Washington, DC: Kaiser Commission on Medicaid and the Uninsured, Kaiser Family Foundation; 2006. Available at: http://www.kff.org/medicaid/upload/ 7490.pdf. Accessed May 10, 2011.

27. Raven MC, Billings JC, Goldfrank LR, Manheimer ED, Gourevitch MN. Medicaid patients at high risk for frequent hospital admission: real-time identification and remediable risks. J Urban Health 2009;86: $230-41$.

28. Zook Cj, Moore FD. High-cost users of medical care. New Engl J Med 1980;302:996-1002.

29. Crane SJ, Tung EE, Hanson GJ, Cha S, Chaudhry $\mathrm{R}$, Takahashi PY. Use of an electronic administrative database to identify older community dwelling adults at high-risk for hospitalization or emergency department visits: the elders risk assessment index. BMC Health Serv Res 2010;10:338.

30. McGill R, Tukey JW, Larsen WA. Variations of box plots. Am Stat 1978;3212-6.

31. Epstein AM, Jha AK, Orav EJ. The relationship between hospital admission rates and rehospitalizations. New Engl J Med 2011;365:2287-95.

32. Bodenheimer T. Coordinating care-a perilous journey through the health care system. New Engl J Med 2008;358:1064-71.

33. Coleman EA, Smith JD, Raha D, Min SJ. Posthospital medication discrepancies: prevalence and contributing factors. Arch Intern Med 2005;165:1842-7. 